\title{
CONSERVATIVE FORMS OF BOLTZMANN'S COLLISION OPERATOR: LANDAU REVISITED
}

\author{
CÉDRIC VILlani ${ }^{1}$
}

\begin{abstract}
We show that Boltzmann's collision operator can be written explicitly in divergence and double divergence forms. These conservative formulations may be of interest for both theoretical and numerical purposes. We give an application to the asymptotics of grazing collisions.
\end{abstract}

AMS Subject Classification. 35Q72, 82C40.

Received: March 19, 1998.

\section{INTRODUCTION}

Boltzmann's collision operator is the main model in statistical physics for describing the interaction between colliding particles. It reads

$$
Q(f, f)=\frac{1}{2} \int_{\mathbb{R}^{N}} d v_{*} \int_{S^{N-1}} d \omega B\left(v-v_{*}, \omega\right)\left(f^{\prime} f_{*}^{\prime}-f f_{*}\right)
$$

where $f: \mathbb{R}^{N} \longrightarrow \mathbb{R}_{+}$is a nonnegative $L^{1}$ function, standing for the distribution of the velocities of the particles,

$$
\left\{\begin{array}{l}
v^{\prime}=v-\left(v-v_{*}, \omega\right) \omega, \\
v_{*}^{\prime}=v_{*}+\left(v-v_{*}, \omega\right) \omega,
\end{array}\right.
$$

and $f^{\prime}=f\left(v^{\prime}\right)$, and so on. As $\omega$ varies in the unit sphere $S^{N-1}, v^{\prime}$ and $v_{*}^{\prime}$ describe all the possible postcollisional velocities of two particles colliding with respective velocity $v$ and $v_{*}$, taking into account that the collisions are assumed to be elastic, i.e.

$$
\left\{\begin{array}{l}
v^{\prime}+v_{*}^{\prime}=v+v_{*}, \\
\left|v^{\prime}\right|^{2}+\left|v_{*}^{\prime}\right|^{2}=|v|^{2}+\left|v_{*}\right|^{2} .
\end{array}\right.
$$

Moreover, $B(z, \omega)$ is a nonnegative weight function for all possible directions of the parameter $\omega$. It is always assumed on physical grounds that $B$ depends only on $|z|$ and $|(z, \omega)|$. A factor $1 / 2$ stands in front of the collisional integral because in the representation (2) the unit sphere is covered twice: alternatively, we could restrict the integration to the set of $\left(v, v_{*}, \omega\right)$ satisfying $\left(v-v_{*}, \omega\right)>0$.

$Q(f, f)$ at point $v$ gives the variation of the "number" of particles with velocity $v$, in a unit of time, due to collisions. It is often split into its positive and negative parts, $Q=Q^{+}-Q^{-}$, which are called respectively the gain and loss terms of the collision operator.

\footnotetext{
${ }^{1}$ École Normale Supérieure, DMI, 45 rue d’Ulm, 75230 Paris Cedex 05, France. e-mail: villani@dmi.ens.fr
} 
Another useful representation of $Q$ is obtained by using a local system of spherical coordinates with axis $v-v_{*}$ and spherical angle $\sigma$ :

$$
Q(f, f)=\int_{\mathbb{R}^{N}} d v_{*} \int_{S^{N-1}} d \sigma \widetilde{B}\left(v-v_{*}, \sigma\right)\left(f^{\prime} f_{*}^{\prime}-f f_{*}\right),
$$

with

$$
\left\{\begin{array}{l}
v^{\prime}=\frac{v+v_{*}}{2}+\frac{\left|v-v_{*}\right|}{2} \sigma \\
v_{*}^{\prime}=\frac{v+v_{*}}{2}+\frac{\left|v-v_{*}\right|}{2} \sigma
\end{array}\right.
$$

and

$$
\widetilde{B}(z, \sigma)=\frac{B(z, \omega)}{2^{N-1}\left|\left(\frac{z}{|z|}, \omega\right)\right|^{N-2}} .
$$

If the interaction between two particles is given by an inverse-power force $1 / r^{s}$ ( $r$ being the distance between particles) with $s \geq 2$, then ( $c f .[1])$

$$
\widetilde{B}(z, \sigma)=|z|^{\gamma} \zeta(\theta)
$$

where $\theta \in[0, \pi]$ is the angle between $z$ and $\sigma$. If $N=3$, then $\gamma=(s-5) /(s-1)$ and $\zeta$ is smooth on $(0, \pi / 2)$, but $\zeta(\theta) \sim C \theta^{-(s+1) /(s-1)}$ with $C>0$ as $\theta \rightarrow 0$. This singularity corresponds to the so-called grazing collisions, i.e. with a very small amount of momentum transfer (equivalently, $\left(v-v_{*}, \omega\right) \simeq 0$ ). Though it is nonintegrable, it is possible to give a distributional sense to $Q(f, f)$ under rather weak assumptions, provided that

$$
\Lambda \equiv \int_{0}^{\pi} d \theta \zeta(\theta) \sin ^{2} \frac{\theta}{2}<\infty
$$

i.e. that the total cross-section for momentum transfer be finite. See [6] and the references therein for a detailed study.

For Coulomb interactions, $s=2$ and the integral (6) diverges logarithmically, due to the effect of long-range interactions. To circumvent this difficulty, Landau [4] formally performed asymptotics in which the grazing collisions become preponderant, and obtained a new collision operator,

$$
Q_{L}(f, f)=\frac{\partial}{\partial v_{i}}\left\{\int_{\mathbb{R}^{N}} d v_{*} a_{i j}\left(v-v_{*}\right)\left(f_{*} \frac{\partial f}{\partial v_{j}}-f \frac{\partial f_{*}}{\partial v_{* j}}\right)\right\}
$$

with

$$
\begin{gathered}
a_{i j}(z)=\Lambda \Psi(|z|) \Pi_{i j}(z), \quad \Pi_{i j}(z)=\delta_{i j}-\frac{z_{i} z_{j}}{|z|^{2}} \\
\Psi(|z|)=|z|^{\gamma+2} \quad\left(=\frac{1}{|z|} \quad \text { for Coulomb potential }\right) .
\end{gathered}
$$

The case $\gamma \neq-3$ is formally treated in [2] for instance. 
Assuming of course $\Lambda$ to be finite, the operator (7) is well-defined and is believed to give a satisfactory description of the collisions in a dilute plasma. The basis of Landau's analysis was to write formally Boltzmann's collision operator in a conservative form,

$$
Q(f, f)=-\nabla_{v} \cdot s
$$

and the $j$ th component of $s$ is given by

$$
s_{j}(v)=-\int_{q_{j}>0} d \omega d v_{*} \int_{v_{j}-q_{j}}^{v_{j}} d w_{j} B\left(w-w_{*}, \omega\right)\left[f(w+q) f\left(w_{*}-q\right)-f(w) f\left(w_{*}\right)\right]
$$

where $q=\left(v-v_{*}, \omega\right) \omega$ is the transferred momentum; see [4], paragraph 41 .

It is easily seen that this expression is a crude approximation of (1), since the flux (9) counts particles going through a hyperplane. But both expressions $(1,8)$ coincide in the limit when all the collisions become grazing, because, due to the small amount of momentum transfer, the velocity of a particle changes continuously - and a small surface element, viewed from very close, looks like a hyperplane. In fact, it is sometimes stated in physics textbooks that the expression (8) is meaningless in the general case, because, contrary to a diffusion process, the velocity of particles does not change continuously (hence particles "jump" in the velocity space $\mathbb{R}^{N}$, and the flux is not well-defined).

However, we shall show that, at least from the mathematical point of view, it is perfectly possible to write the Boltzmann collision operator in a conservative form, even if particles undergo sudden changes of velocity. For instance, one possible expression for the flux of particles is

$$
J(f, f)=-\int_{\left(v-v_{*}, \omega\right)>0} d v_{*} d \omega B\left(v-v_{*}, \omega\right) \int_{0}^{\left(v-v_{*}, \omega\right)} d r f(v+r \omega) f\left(v_{*}+r \omega\right) \omega .
$$

What is more, we shall prove that $Q$ can be written as a double divergence, that is,

$$
Q(f, f)=\sum_{i j} \partial_{i j} A_{i j}(f, f)
$$

These formulas could be interesting for the mathematical study of the Boltzmann equation, especially the singularity in $B$. In particular, we note that in the formula (10) a singularity of order 1 in $B$ has been formally reported on the divergence operation. Indeed, if $f$ is smooth,

$$
\int_{0}^{\left(v-v_{*}, \omega\right)} d r f(v+r \omega) f\left(v_{*}+r \omega\right)=O\left(\left(v-v_{*}, \omega\right)\right) .
$$

On the other hand, the representation (10) gives the flux as an integral operator with one multiplicity more than the collision operator (1), i.e. $2 N$ instead of $2 N-1$. In fact, one can also write $J$ as an expression of the form

$$
J(f, f)=\int_{\mathbb{R}^{2 N}} d v_{*} d v_{\circ} f\left(v_{*}\right) f\left(v_{\circ}\right) D\left(v, v_{*}, v_{\circ}\right) .
$$

Such a formula could be useful for devising new deterministic numerical schemes for the Boltzmann equation. Indeed, the fact that $\omega$ runs through the unit sphere in (1) appears to often cause delicate problems for discretization [3].

The organization of the paper is as follows. In Section 2, we recall the basic identities involving $Q$; then we state several forms of $Q(f, f)$, considered either as a divergence or as a double divergence. We also give a double divergence form for Landau's collision operator (7). These expressions are established in Sections 3 
to 6. Finally, in Section 7, we give an application of the results of Section 3: a simplified proof of a result by Desvillettes [2] that if a fixed function $f$ is smooth enough, then in a suitable grazing asymptotics process, $Q(f, f)$ reduces to $Q_{L}(f, f)$.

\section{BASIC IDENTITIES AND MAIN RESUltS}

We first recall some well-known facts about Boltzmann's collision operator. Let $\varphi$ be a smooth test function; we consider

$$
\int Q(f, f) \varphi=\int d v d v_{*} d \sigma \widetilde{B}\left(v-v_{*}, \sigma\right)\left(f^{\prime} f_{*}^{\prime}-f f_{*}\right) \varphi(v) .
$$

By the change of variables (involutive and with unit Jacobian) $\left(v, v_{*}\right) \rightarrow\left(v^{\prime}, v_{*}^{\prime}\right)$, noting that $\widetilde{B}\left(v^{\prime}-v_{*}^{\prime}, \sigma\right)=$ $\widetilde{B}\left(v-v_{*}, \sigma\right)$, we obtain

$$
\int Q(f, f) \varphi=\int d v d v_{*} d \sigma \widetilde{B} f f_{*}\left(\varphi^{\prime}-\varphi\right)
$$

where we have omitted for simplicity the arguments of $\widetilde{B}$. This is Maxwell's form [5] of Boltzmann's collision operator. Now, by the change of variables $\left(v, v_{*}\right) \rightarrow\left(v_{*}, v\right)$,

$$
\int Q(f, f) \varphi=\frac{1}{2} \int d v d v_{*} d \sigma \widetilde{B} f f_{*}\left(\varphi^{\prime}+\varphi_{*}^{\prime}-\varphi-\varphi_{*}\right) .
$$

This formula can actually be taken as a definition of $Q(f, f)$ in the sense of distributions [6], as soon as, say, $\int f(v)\left(1+|v|^{2+\gamma}\right) d v<\infty$ and $\widetilde{B}(z, \sigma) \sin ^{N-2_{\theta}}=\Phi(|z|) \zeta(\theta)$ with $\Phi(|z|) \leq C\left(1+|z|^{\gamma}\right)$ and $\int \theta^{2} \zeta(\theta) d \theta<\infty$. It is also clear that analogous formulas hold for the representation (1).

Similarly ( $c f .[6])$, for Landau's collision operator

$$
\int Q_{L}(f, f) \varphi=\frac{1}{2} \int d v d v_{*} f f_{*} a_{i j}\left(v-v_{*}\right)\left(\partial_{i j} \varphi+\left(\partial_{i j} \varphi\right)_{*}\right)+\int d v d v_{*} f f_{*} b_{i}\left(v-v_{*}\right)\left(\partial_{i} \varphi-\left(\partial_{i} \varphi\right)_{*}\right),
$$

with $b_{i}(z)=\sum_{j} \partial_{j} a_{i j}(z)$

In view of these formulas and of the conservation laws (3),

$$
\int Q(f, f)\left(\begin{array}{c}
1 \\
v_{i} \\
|v|^{2}
\end{array}\right)=0 ; \quad \int Q_{L}(f, f)\left(\begin{array}{c}
1 \\
v_{i} \\
|v|^{2}
\end{array}\right)=0
$$

This alone suffices to prove that $Q$ (resp. $Q_{L}$ ) is formally a double divergence. Indeed, denoting by $\widehat{Q}(\xi)$ the Fourier transform of $Q(f, f)$, we see that

$$
\widehat{Q}(0)=0, \quad \frac{\partial \widehat{Q}}{\partial \xi_{i}}(0)=0, \quad \Delta_{\xi} \widehat{Q}(0)=0
$$

Therefore, as soon as $\widehat{Q}$ is $C^{2}$,

$$
\widehat{Q}(\xi)=\widehat{Q}(0)+\xi \cdot \frac{\partial \widehat{Q}}{\partial \xi}(0)+\int_{0}^{1} D^{2} \widehat{Q}(t \xi, t \xi) \cdot(\xi, \xi)(1-t) d t=\sum_{i j} \alpha_{i j} \xi_{i} \xi_{j}
$$


with $\alpha_{i j}(\xi)=\int_{0}^{1} \partial_{i j} \widehat{Q}(t \xi, t \xi)(1-t) d t$. This is exactly the Fourier version of (11). Moreover, we note that $\sum_{i} \alpha_{i i}(0)=0$, which means that

$$
\int \sum_{i} A_{i i}(f, f)=0
$$

But the remarkable feature of Boltzmann's collision operator is that we shall be able to obtain explicit expressions in both representations $(1,4)$, whose physical meaning will be clear.

We note that the condition $\int X=0$ is definitely not necessary for $X$ to be the divergence of some flux $J$. Indeed, the flux could be nonvanishing at infinity, in which case it is impossible to invoke Stokes' formula and conclude that $\int X=0$ (such an example will be seen in Sect. 6). However, for both mathematical and numerical purposes, it seems desirable to impose that $J \longrightarrow 0$ at infinity.

Theorem 1. In the sense of distributions, $Q(f, f)=-\nabla \cdot J(f, f)$ with

$$
\begin{aligned}
J(f, f) & =J=-\int_{\left(v-v_{*}, \omega\right)>0} d v_{*} d \omega B\left(v-v_{*}, \omega\right) \int_{0}^{\left(v-v_{*}, \omega\right)} d r f(v+r \omega) f\left(v_{*}+r \omega\right) \omega \\
& =\int_{\left(v_{*}-v, v_{\circ}-v\right)<0} d v_{\circ} d v_{*} f\left(v_{\circ}\right) f\left(v_{*}\right) B\left(v_{\circ}-v_{*}, \frac{v-v_{\circ}}{\left|v-v_{\circ}\right|}\right) \frac{v_{-} v_{\circ}}{\left|v-v_{\circ}\right|^{N}} .
\end{aligned}
$$

Alternatively,

$$
\begin{aligned}
J(f, f)=\widetilde{J}=-\int d v_{*} d \sigma \widetilde{B}\left(v-v_{*}, \sigma\right) \int_{0}^{\frac{\left|v-v_{*}\right|}{2}} d r & \\
\left\{f\left(v+r \frac{v-v_{*}}{\left|v-v_{*}\right|}\right) f\left(v_{*}+r \frac{v-v_{*}}{\left|v-v_{*}\right|}\right) \frac{v-v_{*}}{\left|v-v_{*}\right|}\right. & \left.-f\left(v-r \sigma-\frac{v-v_{*}}{2}\right) f\left(v-r \sigma+\frac{v-v_{*}}{2}\right) \sigma\right\} .
\end{aligned}
$$

\section{Remarks}

1. The expressions (14) and (16) differ by a divergence-free vector field, but need not be equal to each other.

2. Both expressions go to 0 at infinity under suitable assumptions on $f$ and $B$, as is most conveniently seen by formula (15): for instance, if $f$ has compact support and $B$ is bounded, the decay as $|v| \rightarrow \infty$ is at least like $C /|v|$ for some $C>0$.

3. Several variants are given in the paper. In particular, we also give a representation of (16) in the form (12).

With this theorem at hand, it is very easy to perform the asymptotics of grazing collisions, and show that $J$ converges towards the expression in curly brackets in (7). This will be done in Section 7 .

As we said before, a double divergence formula is also available for the operator (1).

Theorem 2. In the sense of distributions, $Q(f, f)=\sum_{i k} \partial_{i k} A_{i k}(f, f)$, with

$$
\begin{aligned}
A_{i k}=-\int d v_{*} d \sigma \widetilde{B}\left(v-v_{*}, \sigma\right) & \int_{0}^{\frac{\left|v-v_{*}\right|}{2}} d r \\
& \begin{aligned}
&\left\{f\left(v+r \frac{v-v_{*}}{\left|v-v_{*}\right|}\right) f\left(v_{*}+r \frac{v-v_{*}}{\left|v-v_{*}\right|}\right) r \frac{\left(v-v_{*}\right)_{k}\left(v-v_{*}\right)_{i}}{\left|v-v_{*}\right|^{2}}\right. \\
& \quad\left.\quad f\left(v-r \sigma-\frac{v-v_{*}}{2}\right) f\left(v-r \sigma+\frac{v-v_{*}}{2}\right)\left(\frac{\left|v-v_{*}\right|}{2}-r\right) \sigma_{i} \sigma_{k}\right\} .
\end{aligned}
\end{aligned}
$$




\section{Remark}

In addition, $\sum A_{i i}(f, f)$ is itself a divergence! We shall not give an explicit expression for this, but show how to obtain it.

By the way, we shall see that Landau's collision operator (already in divergence form) is also a double divergence.

The expressions in Theorems 1 and 2 are first guessed by formal physical reasonings; then we check their mathematical correctness. The first expression in Theorem 1 is established in Section 3 by an explicit computation of the "flux" of particles in velocity space. Since the notion of flux is not well-defined for a process with sudden jumps, we simply assume that particles follow straight lines in the velocity space.

We recall that the flux $j$ of some quantity $n$ in $\mathbb{R}^{N}$ is formally defined by $d n=j \cdot d \vec{S} d \tau$, giving the (algebraic) amount of quantity $n$ going through an infinitesimal surface $d \vec{S}$ in a time $d \tau$. In other words, if $S$ is a closed surface enclosing a volume $V$, then the variation of $\int_{V} d n$ is given by $-\int_{S} j \cdot d \vec{S}$, if $d \vec{S}$ denotes the outward normal to $S$. By Stokes' formula, this is also $-\int_{V} \nabla \cdot j$. Taking $V$ to be an infinitesimal volume $d v$ in velocity space, and $n$ the number of particles, we get

$$
Q(f, f) d v=\frac{d n}{d \tau}=-(\nabla \cdot j) d v
$$

We make it clear that this derivation must only be taken to be a heuristic one, and that we shall give rigorous proofs of the corresponding formulas.

To express $Q(f, f)$ as a double divergence, it suffices to obtain a divergence form for $Q(f, f)$ and $Q(f, f) v_{i}$, $1 \leq i \leq N$, as shown in Section 4. But if we try to compute the flux of $Q(f, f) v_{i}$ in the same manner as before (with straight pathlines), this leads immediately to definite contradictions; therefore, we have to develop a more complicated scheme, based on the $\sigma$-representation. The final expressions are obtained in Section 5 . As for the Landau equation, we do not know of any convenient representation of $Q_{L}$ in terms of flux, but it will be easy to obtain conservative representations, either by a direct method, or by analogy with the study of the Boltzmann operator.

\section{FiRST CONSERVATIVE FORM}

In this section, we use the representation (1). Let $v$ be a fixed velocity in the phase space $\mathbb{R}^{N}$, and let $\vec{e}_{1}$ be a fixed unit vector, say from left to right. We want to compute the component $j_{1}$ along $\vec{e}_{1}$ of the collisional flux of particles $j$ at point $v$. In order to do so, we consider an infinitesimal surface $d S$ with center $v$, perpendicular to $\vec{e}_{1}$, and we count all particles going from one side of $d S$ to the other, due to collisions. Let $v_{\circ}$ denote the velocity of an arbitrary particle $P_{\circ}$, which may go through $d S$, and $v_{*}$ denote the velocity of a test-particle $P_{*}$ which may encounter the previous particle. Furthermore, let $q$ be the momentum transferred from $P_{*}$ to $P_{\circ}$ : $\left.q=\left(v_{*}-v_{\circ}, \omega\right) \omega\right)$. By definition, a particle with velocity $v_{\circ}$ can go through $d S$ only if $v \in\left[v_{\circ}, v_{\circ}+q\right]$, which implies that the collisional parameter $\omega$ is determined by

$$
\omega=\omega_{c}=\frac{v_{\circ}-v}{\left|v_{\circ}-v\right|}
$$

(we do not take into account the other possibility that $\omega=-\omega_{c}$, since this would result in a double covering of the sphere, and we do not consider the exceptional event $v_{\circ}=v$ ).

Now, the number of collisions in which a particle $P_{\circ}$ with velocity $v_{\circ}$ encounters $P_{*}$ with velocity $v_{*}$, such that the collision parameter is $\omega_{c}$, is

$$
d v_{*} d v_{\circ} f\left(v_{*}\right) f\left(v_{\circ}\right) B\left(v_{\circ}-v_{*}, \frac{v_{\circ}-v}{\left|v_{\circ}-v\right|}\right) d \omega
$$



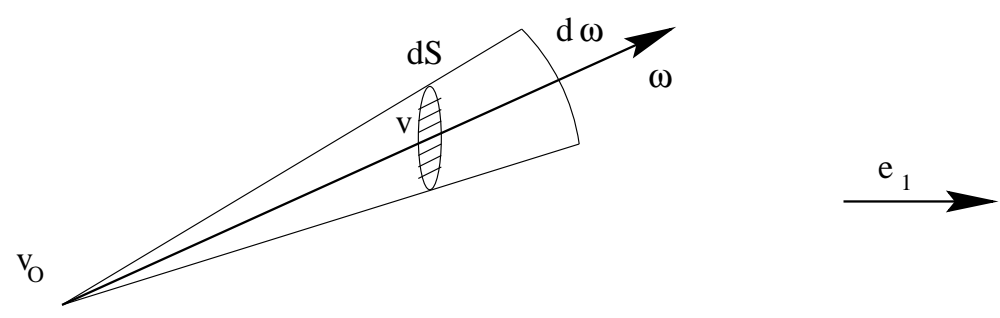

FiguRE 1. Particle going through a surface element in velocity space

where $d \omega$ is the solid angle with which $d S$ is viewed from $v_{\circ}$. By an easy calculation in elementary geometry,

$$
d \omega=\frac{d S\left|\cos \left(\omega_{c}, \vec{e}_{1}\right)\right|}{\left|v_{\circ}-v\right|^{N-1}}
$$

Since

$$
\cos \left(\omega_{c}, \vec{e}_{1}\right)=\left(\omega_{c}\right)_{1}=\frac{v_{\circ 1}-v_{1}}{\left|v_{\circ}-v\right|}
$$

and since the first component $j_{1}$ of the flux is obtained by dividing the algebraic number of particles going through the element of surface by the value of $d S$, we obtain

$$
\begin{aligned}
j_{1}=\iint_{I^{+}} d v_{*} d v_{\circ} f\left(v_{*}\right) f\left(v_{\circ}\right) B & \left(v_{\circ}-v_{*}, \frac{v_{\circ}-v}{\left|v_{\circ}-v\right|}\right) \frac{\left|v_{\circ}-v_{1}\right|}{\left|v_{\circ}-v\right|} \frac{1}{\left|v_{\circ}-v\right|^{N-1}} \\
& -\iint_{I^{-}} d v_{*} d v_{\circ} f\left(v_{*}\right) f\left(v_{\circ}\right) B\left(v_{\circ}-v_{*}, \frac{v_{\circ}-v}{\left|v_{\circ}-v\right|}\right) \frac{\left|v_{\circ}-v_{1}\right|}{\left|v_{\circ}-v\right|} \frac{1}{\left|v_{\circ}-v\right|^{N-1}},
\end{aligned}
$$

where $I^{+}$(resp. $\left.I^{-}\right)$is the set of all couples $\left(v_{\circ}, v_{*}\right)$ such that, in the collision of $P_{\circ}$ and $P_{*}, P_{\circ}$ goes through $d S$ from the left to the right (resp. from the right to the left), i.e.

$$
I^{+}=\left\{\left(v_{\circ}, v_{*}\right) \in \mathbb{R}^{2 N} / v_{1}-q_{1}<v_{\circ 1}<v_{1}\right\}, \quad I^{-}=\left\{\left(v_{\circ}, v_{*}\right) \in \mathbb{R}^{2 N} / v_{1}-q_{1}>v_{\circ 1}>v_{1}\right\} .
$$

The expression (18) is the desired quantity. In order to obtain better expressions for $I^{+}$and $I^{-}$, let us write

$$
v_{\circ}=v+r \omega, \quad r \geq 0, \quad \omega \in S^{N-1} .
$$

In these variables, by (2) we easily find

$$
I^{+}=\left\{\omega_{1}<0, \quad\left(v-v_{*}, \omega\right)>0\right\}, \quad I^{-}=\left\{\omega_{1}>0, \quad\left(v-v_{*}, \omega\right)>0\right\}
$$

Since $\left|\omega_{1}\right| 1_{\omega_{1}<0}-\left|\omega_{1}\right| 1_{\omega_{1}>0}=-\omega_{1}$, and since $\left(v-v_{*}, \omega\right)$ has the same sign as $\left(v-v_{*}, v_{\circ}-v\right)$, (18) can be rewritten as

$$
j_{1}=\iint_{\left(v_{*}-v, v_{\circ}-v\right)<0} d v_{*} d v_{\circ} f\left(v_{*}\right) f\left(v_{\circ}\right) B\left(v_{\circ}-v_{*}, \frac{v-v_{\circ}}{\left|v-v_{\circ}\right|}\right) \frac{v_{1}-v_{\circ}}{\left|v-v_{\circ}\right|} \frac{1}{\left|v-v_{\circ}\right|^{N-1}} .
$$

This leads us to a first possible expression of the flux, which is formula (15). 
To show that the expressions in $(14,15)$ are equal, we use the spherical change of variables with center $v$ which is given by (19).

Let us set $A=I^{+} \cup I^{-}=\left\{\left(v-v_{*}, \omega\right)>0\right\}$. Since $d v_{\circ}=r^{N-1} d r d \omega$,

$$
j_{1}=-\int_{A} d r d \omega d v_{*} f\left(v_{*}\right) f(v+r \omega) B\left(v+r \omega-v_{*}, \omega\right) \omega_{1}
$$

As a consequence, the value of the flux of particles at point $v$ can also be written as

$$
j=-\iint_{A} d r d \omega d v_{*} f\left(v_{*}\right) f(v+r \omega) B\left(v+r \omega-v_{*}, \omega\right) \omega=J(f, f) .
$$

This ends the first part of our program, namely guess the flux of particles by formal reasonings. We now proceed to compute the divergence of $J(f, f)$, and show that it is actually equal to $-Q(f, f)$. We begin with formal manipulations, and then show how to give them a quite rigorous and simple meaning in distributional sense, by using the weak formulation of $Q$.

Let us differentiate the opposite of (20) with respect to $v_{1}$. This gives

$$
\int d r d \omega \int_{\partial A} f\left(v_{*}\right) f(v+r \omega) B\left(v+r \omega-v_{*}, \omega\right) \omega_{1}^{2}+\int d r d \omega \int_{A} d v_{*} f\left(v_{*}\right) \omega_{1} \partial_{1}\left[f(v+r \omega) B\left(v+r \omega-v_{*}, \omega\right)\right]
$$

with $\partial A=\left\{\left(v-v_{*}, \omega\right)=0\right\}$. Since $\omega_{1}^{2}+\cdots+\omega_{N}^{2}=1$ and $\omega_{1} \partial_{1}+\cdots+\omega_{N} \partial_{N}=\omega \cdot \nabla$, we get

$$
-\nabla \cdot J=(I)+(I I)
$$

with

$$
(I)=\int d \omega \int_{\left(v-v_{*}, \omega\right)=0} d r d \tilde{v}_{*} f\left(v_{*}\right) f(v+r \omega) B\left(v+r \omega-v_{*}, \omega\right)
$$

where $\tilde{v}_{*}=v_{*}^{\perp}$ is the component of $v_{*}$ which is orthogonal to $\omega$, and

$$
(I I)=\int d r d \omega \int_{A} d v_{*} f\left(v_{*}\right) \omega \cdot \nabla_{v}\left[f(v+r \omega) B\left(v+r \omega-v_{*}, \omega\right)\right] .
$$

As for $(I)$, let us introduce the new variable $w_{*}$ such that, in the collision of particles with velocities $v$ and $w_{*}$,

$$
\left\{\begin{array}{lll}
w_{*}^{\prime}=w_{*}+\left(v-w_{*}, \omega\right) \omega & =v_{*} \\
v^{\prime}=v-\left(v-w_{*}, \omega\right) \omega & =v+r \omega
\end{array}\right.
$$

i.e. $w_{*}^{\perp}=v_{*}^{\perp}$ and $\left(w_{*}, \omega\right)=(v, \omega)+r$. For fixed $\omega$ and $v$, the change of variables $\left(\tilde{v}_{*}, r\right) \rightarrow w_{*}=\left(\tilde{v}_{*}, r+(v, \omega)\right)$ is a translation. Moreover, $w_{*}$ varies in the half-space $\mathbb{R}^{N-1} \times[(v, \omega),+\infty)$. After the use of the symmetry $(r, \omega) \rightarrow(-r,-\omega)$, which leaves the integrand of $(I)$ unchanged and allows integration on all space $\mathbb{R}^{N}$, we finally obtain

$$
(I)=\frac{1}{2} \int d \omega d w_{*} f\left(w_{*}^{\prime}\right) f\left(v^{\prime}\right) B\left(v^{\prime}-w_{*}^{\prime}, \omega\right) .
$$

Since $B\left(v^{\prime}-w_{*}^{\prime}, \omega\right)=B\left(v-w_{*}, \omega\right)$, this is exactly the "gain term" in (1). 
As for $(I I)$, we note that the action of $\omega \cdot \nabla_{v}$ on a function of $v+r \omega$ is the same as that of $\partial_{r}$, so that it can be rewritten as

$$
\begin{aligned}
& \int d r d \omega \int_{A} d v_{*} f\left(v_{*}\right) \partial_{r}\left[f(v+r \omega) B\left(v+r \omega-v_{*}, \omega\right)\right] \\
= & \int d r \partial_{r}\left[\int_{A} d \omega d v_{*} f\left(v_{*}\right) f(v+r \omega) B\left(v+r \omega-v_{*}, \omega\right)\right] .
\end{aligned}
$$

Using the formula $\int d r \partial_{r} \varphi(r)=-\varphi(0)$ for $\varphi$ vanishing at infinity, we obtain

$$
(I I)=-\int_{A} d \omega d v_{*} f\left(v_{*}\right) f(v) B\left(v-v_{*}, \omega\right) .
$$

By the change of variables $\omega \rightarrow-\omega$, we see that this is exactly the "loss term" in (1).

To obtain a rigorous justification of these manipulations, it suffices to perform them in weak form. Let $\varphi$ be a smooth function with compact support in velocity space. Then,

$$
\begin{aligned}
\int J \cdot \nabla \varphi & =-\int d v \int_{A} d r d v_{*} d \omega f\left(v_{*}\right) f(v+r \omega) B\left(v-v_{*}+r \omega, \omega\right)[\omega \cdot \nabla \varphi(v)] \\
& =-\int_{\left(\bar{v}-v_{*}, \omega\right)>r} d \bar{v} d r d v_{*} d \omega f\left(v_{*}\right) f(\bar{v}) B\left(\bar{v}-v_{*}, \omega\right)[\omega \cdot \nabla \varphi(\bar{v}-r \omega)]
\end{aligned}
$$

where we have used the change of variables $\bar{v}=v+r \omega$. Now, we exchange the variables $\bar{v}$ and $v_{*}$ to get

$$
\int J \cdot \nabla \varphi=-\int_{\left(\bar{v}-v_{*}, \omega\right)<-r} d \bar{v} d r d v_{*} d \omega f\left(v_{*}\right) f(\bar{v}) B\left(\bar{v}-v_{*}, \omega\right)\left[\omega \cdot \nabla \varphi\left(v_{*}-r \omega\right)\right] .
$$

Applying the change of variable $\omega \rightarrow-\omega$ to this last expression, we get

$$
\int J \cdot \nabla \varphi=\int_{\left(\bar{v}-v_{*}, \omega\right)>r} d \bar{v} d r d v_{*} d \omega f\left(v_{*}\right) f(\bar{v}) B\left(\bar{v}-v_{*}, \omega\right)\left[\omega \cdot \nabla \varphi\left(v_{*}+r \omega\right)\right] .
$$

Summing up (21) and (22), and replacing as above $\omega \cdot \nabla$ by $\pm \partial_{r}$, we obtain

$$
\begin{aligned}
\int J \cdot \nabla \varphi & =\frac{1}{2} \int_{\left(v-v_{*}, \omega\right)>r} d v d r d v_{*} d \omega f\left(v_{*}\right) f(v) B\left(v-v_{*}, \omega\right) \partial_{r}\left[\varphi(v-r \omega)+\varphi\left(v_{*}+r \omega\right)\right] \\
& =\frac{1}{2} \int_{A} d v d v_{*} d \omega f(v) f\left(v_{*}\right) B\left(v-v_{*}, \omega\right) \int_{0}^{\left(v-v_{*}, \omega\right)} d r \partial_{r}\left[\varphi(v-r \omega)+\varphi\left(v_{*}+r \omega\right)\right] \\
& =\frac{1}{2} \int_{A} d v d v_{*} d \omega f(v) f\left(v_{*}\right) B\left(v-v_{*}, \omega\right)\left[\varphi\left(v_{*}^{\prime}\right)-\varphi\left(v_{*}\right)+\varphi\left(v^{\prime}\right)-\varphi(v)\right] \\
& =\frac{1}{4} \int d v d v_{*} d \omega f f_{*} B\left(v-v_{*}, \omega\right)\left[\varphi^{\prime}+\varphi_{*}^{\prime}-\varphi-\varphi_{*}\right] \\
& =\int Q(f, f) \varphi .
\end{aligned}
$$

This proves that, in distributional sense, $Q=-\nabla \cdot J$.

Finally, we note that the expression of Theorem 1 is obtained by applying to (20) the change of variables $v_{*} \rightarrow v_{*}-r \omega$. 


\section{How TO GO FURTHER}

Now, we would like to obtain a double divergence form of $Q$. First note that, since $\int Q v_{k}=0$ for $1 \leq k \leq N$, $Q v_{k}$ should also be a divergence. Let us assume that we know how to compute a suitable flux $J^{k}$ for it, so that

$$
\left\{\begin{array}{l}
Q=-\nabla \cdot J \\
Q v_{k}=-\nabla \cdot J^{k}
\end{array}\right.
$$

Then

$$
-\nabla \cdot\left(J v_{k}\right)=-(\nabla \cdot J) v_{k}-J_{k}=Q v_{k}-J_{k}=-\nabla \cdot J^{k}-J_{k},
$$

so that

$$
J_{k}=-\nabla \cdot\left(J^{k}-J v_{k}\right)=-\sum_{i} \partial_{i}\left(J_{i}^{k}-J_{i} v_{k}\right)
$$

Therefore, $Q=\sum \partial_{i k} A_{i k}$ with

$$
A_{i k}=J_{i}^{k}-J_{i} v_{k}
$$

A little more can be said. Suppose that we know how to compute an energy flux $J^{E}$, i.e.

$$
Q|v|^{2}=-\nabla \cdot J^{E}
$$

Then

$$
-\nabla \cdot J^{E}=Q|v|^{2}=\sum_{i} Q v_{i} v_{i}=-\sum_{i}\left(\nabla \cdot J^{i}\right) v_{i}=-\sum_{i} \nabla \cdot\left(J^{i} v_{i}\right)+\sum_{i} J_{i}^{i}
$$

so that

$$
\sum_{i} J_{i}^{i}=-\nabla \cdot J^{E}+\sum_{i} \nabla \cdot\left(J^{i} v_{i}\right)=-\nabla \cdot\left(J^{E}-\sum_{i} J^{i} v_{i}\right)
$$

In other words, $\sum A_{i i}$ is itself a divergence.

Therefore, we see that the only matter is to compute the flux of $v_{k}$ and $v^{2}$, that is, the collisional invariants $C(v)$.

If we still assume that particles go along straight pathlines, a serious problem arises: what value should be given to $C(v)$, while the particle lies between $v_{\circ}$ and $v_{\circ}+q$ ? In fact it is impossible to use this scheme, because such a "flux" would not be conservative, in the following sense. Let $S$ be a surface enclosing a volume $V$; let $v_{\circ}$ be inside $V$, and $v_{*}$ outside. Then it may happen that $v_{\circ}^{\prime}$ be also inside $V$, yet $v_{*}^{\prime}$ remain outside. Thus, the particles may interact, and momentum may be transferred from the exterior to the interior of $V$, without any momentum crossing the surface $S$.

The remedy to this pathology is simply to use the representation (4), and the following underlying scheme. Consider two colliding particles $P_{\circ}$ and $P_{*}$ with velocities $v_{\circ}$ and $v_{*}$, and $C(v)$ the collisional invariant whose flux we want to compute. During the collision, we clearly distinguish between the position of the particles in the phase space, say $\xi_{0}, \xi_{*}$, and the quantities $C\left(v_{\circ}\right), C\left(v_{*}\right)$, etc., which are attached to them.

1. At the beginning of the collision, $\xi_{\circ}=v_{\circ}, \xi_{*}=v_{*}$. Then the two particles converge in straight lines towards the center point in the phase space, $\xi_{\#}=\left(v_{\circ}+v_{*}\right) / 2$. $C$ is unchanged for both particles. 


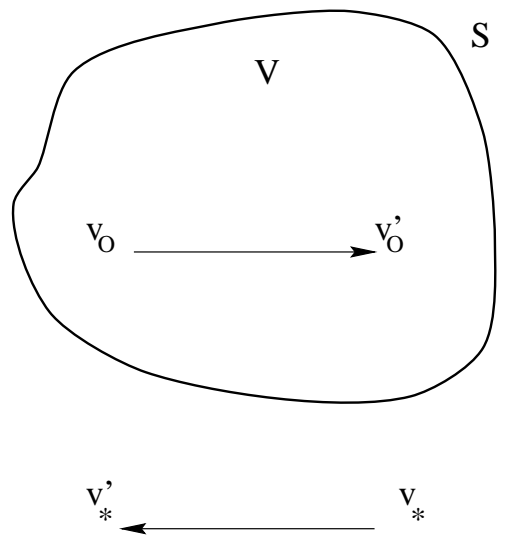

Figure 2. This collision makes the quantity of momentum inside $V$ change, yet no particle has gone through the surface $S$.

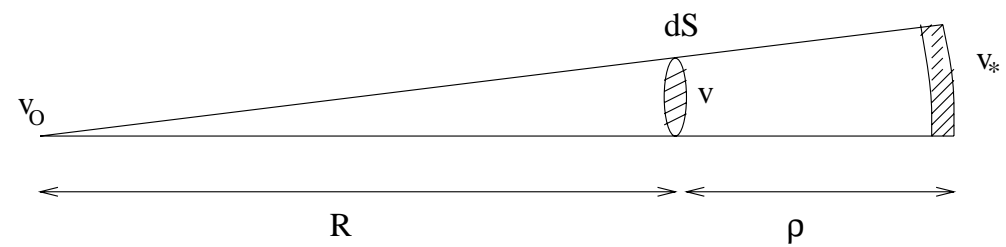

Figure 3. First step of the collisional scheme

2. Once both particles are at the same position $\xi_{\#}$, occurs the momentum transfer: $C\left(v_{\circ}\right)$ and $C\left(v_{*}\right)$ are replaced by $C\left(v_{\circ}^{\prime}\right)$ and $C\left(v_{*}^{\prime}\right)$, respectively. This step does not contribute to any flux of $C$, since $C$ is a collisional invariant.

3. Then, the particles go in straight paths towards their final positions, $\xi_{\circ}^{\prime}=v_{\circ}^{\prime}$ and $\xi_{*}^{\prime}=v_{*}^{\prime}$, without any change of $C$.

It now remains to adapt the method of Section 3 to this scheme. We note that step 2 definitely fails for functions of the velocity that are not collision invariants.

\section{Computing in $\sigma$-RePRESEntation}

\section{First step of the scheme}

We proceed to compute the flux of particles implied by the first step of the above collisional scheme. As before, we fix an infinitesimal surface $d S$ with center $v$, and a unit vector $\vec{e}_{1}$ perpendicular to $d S$. Clearly, $v_{*}, v_{\circ}$ and $v$ must be aligned for the flux to be non $0 ; v$ must even lie between $v_{*}$ and $v_{\circ}$. Therefore, we put

$$
\left\{\begin{array}{l}
v_{*}=v+\rho \beta, \\
v_{\circ}=v-R \beta
\end{array}\right.
$$

with $\rho, R \geq 0$ and $\beta \in S^{N-1}$.

For fixed $v_{\circ}$, the volume of suitable $v_{*}$, i.e. such that $\left[v_{\circ}, v_{*}\right]$ intersect $d S$, is

$$
d S \frac{\left|\beta_{1}\right|}{R^{N-1}}(\rho+R)^{N-1} d \rho .
$$


Setting

$$
A\left(v_{\circ}-v_{*}\right)=\int d \sigma \widetilde{B}\left(v_{\circ}-v_{*}, \sigma\right),
$$

and using the notation $\int_{X-Y}=\int_{X}-\int_{Y}$, we obtain that the first component of the flux through $d S$ is

$$
j_{1}=\int_{I^{+} I^{-}} d v_{\circ} A\left(v_{\circ}-v_{*}\right) f\left(v_{\circ}\right) f\left(v_{*}\right) \frac{\left|\beta_{1}\right|}{R^{N-1}}(\rho+R)^{N-1} d \rho,
$$

where $I^{+}$(resp. $I^{-}$) denotes the set of velocities $v_{0}$ such that the corresponding particle $P_{0}$ goes through $d S$ from the left to the right.

We can transform this expression by the changes of variables

$$
v_{\circ}=v-R \beta, \quad r=\rho+R, \quad w_{*}=v+r \beta,
$$

so that $w_{*}-v=v_{*}-v_{\circ}$, and

$$
d v_{\circ} d \rho \frac{(\rho+R)^{N-1}}{R^{N-1}}=d R d \rho d \beta(\rho+R)^{N-1}=d r d \rho d \beta r^{N-1}=d w_{*} d \beta .
$$

We determine $I^{+}$and $I^{-}$: the first of these subsets corresponds to $v_{\circ 1}<v_{1}$ and $v_{\# 1}=\left(v_{\circ 1}+v_{* 1}\right) / 2>v_{1}$, i.e. $\beta_{1}>0$ and $\rho>R$. Since $\rho=\left|v-w_{*}\right|-R$, this last condition is $R<\left|v-w_{*}\right| / 2$. For $I^{-}$, it suffices to change the sign of $\beta_{1}$.

Finally, the flux corresponding to the first step of the collisional scheme can be written as

$$
\begin{aligned}
j^{(a)} & =\int d w_{*} \int d \sigma \widetilde{B}\left(v-w_{*}, \sigma\right) \int_{0}^{\frac{\left|v-v_{*}\right|}{2}} d R f(v-R \beta) f\left(w_{*}-R \beta\right) \beta \\
& =\int d w_{*} \int d \sigma \widetilde{B}\left(v-w_{*}, \sigma\right) \int_{0}^{\frac{\left|v-v_{*}\right|}{2}} d R f\left(v+R \frac{v-v_{*}}{\left|v-v_{*}\right|}\right) f\left(w_{*}+R \frac{v-v_{*}}{\left|v-v_{*}\right|}\right) .
\end{aligned}
$$

Accordingly, the corresponding flux for the quantity $C(v)$ is

$$
j^{(a)}(C)=\int d w_{*} \int d_{\sigma} \widetilde{B}\left(v-w_{*}, \sigma\right) \int_{0}^{\frac{\left|v-v_{*}\right|}{2}} d R f\left(v+R \frac{v-v_{*}}{\left|v-v_{*}\right|}\right) f\left(w_{*}+R \frac{v-v_{*}}{\left|v-v_{*}\right|}\right) C\left(v+R \frac{v-v_{*}}{\left|v-v_{*}\right|}\right) .
$$

\section{Remark}

To obtain an expression of the form (12), one can use the exchange of variables $\sigma$ and $\beta$, in the same way as we shall do in the third step of the scheme.

Conversely, we proceed now to compute the divergence of (25). Writing for short $A(r \beta)=A(r)$, we start from

$$
-j_{1}=-\int_{R<\rho} d R d \beta d \rho A(\rho+R) f(v-R \beta) f(v+\rho \beta) \beta_{1}(\rho+R)^{N-1},
$$

and differentiate this expression with respect to $v_{1}$ :

$$
-\partial_{1} j_{1}=-\int_{R<\rho} d R d \beta d \rho A(\rho+R) \beta_{1} \partial_{1}[f(v-R \beta) f(v+\rho \beta)](\rho+R)^{N-1} .
$$


But

$$
\beta \cdot \nabla[f(v-R \beta) f(v+\rho \beta)]=-\partial_{R}[f(v-R \beta)] f(v+\rho \beta)+f(v-R \beta) \partial_{\rho}[f(v+\rho \beta)] .
$$

Therefore, integrating by parts with respect to $\rho$,

$$
\begin{aligned}
-\nabla \cdot j=\int_{R<\rho} d \rho d \beta d R A(\rho+R)(\rho+R)^{N-1} \partial_{R}[f(v-R \beta)] f(v+\rho \beta) & \\
& +\int_{R<\rho} d \rho d \beta d R \partial_{\rho}\left[A(\rho+R)(\rho+R)^{N-1}\right] f(v-R \beta) f(v+\rho \beta),
\end{aligned}
$$

since the boundary terms for $\rho=0$ and $\rho \rightarrow \infty$ both vanish. In the last integral, one can replace $\partial_{\rho}$ by $\partial_{R}$, and obtain

$$
\begin{aligned}
\int d \rho d \beta & \int_{0}^{\rho} d R \partial_{R}\left\{A(\rho+R)(\rho+R)^{N-1} f(v-R \beta) f(v+\rho \beta)\right\} \\
& =\int d \rho d \beta\left[A(\rho+R)(\rho+R)^{N-1} f(v-R \beta) f(v+\rho \beta)\right]_{R=0}^{R=\rho} \\
& =\int d \rho d \beta A(2 \rho)(2 \rho)^{N-1} f(v-\rho \beta) f(v+\rho \beta) \\
& \quad-\int d \rho d \beta A(\rho) \rho^{N-1} f(v) f(v+\rho \beta) \\
& \equiv(\text { Ia })-(\text { IIa }) .
\end{aligned}
$$

Setting $v_{*}=v+\rho \beta$, we see that the last integral (IIa) is

$$
f(v) \int d v_{*} A\left(v-v_{*}\right) f\left(v_{*}\right)
$$

which is the loss term of Boltzmann's operator.

If we perform the computation with (26), we obtain in the end

$$
\begin{gathered}
-\nabla \cdot j^{(a)}(C)=\int d \rho d \beta A(2 \rho)(2 \rho)^{N-1} f(v-\rho \beta) f(v+\rho \beta) C(v-\rho \beta)-\int d \rho d \beta A(\rho) \rho^{N-1} f(v) f(v+\rho \beta) C(v), \\
\equiv(\mathrm{Ia})(C)-(\mathrm{IIa})(C),
\end{gathered}
$$

and the last integral (IIa) $(C)$ is really $Q^{-}(f, f) C(v)$. We note that for $C(v)=v_{k}$, the first integral (Ia) $\left(v_{k}\right)$ is simply

$$
\int d \rho d \beta A(2 \rho)(2 \rho)^{N-1} f(v-\rho \beta) f(v+\rho \beta) v_{k}
$$

indeed, the additional term with $\rho \beta_{k}$ vanishes, as can be seen by the change of variables $\beta \rightarrow-\beta$, which leaves $f(v-\rho \beta) f(v+\rho \beta)$ invariant.

\section{Third step of the scheme}

The computation of the flux attached to the third step is immediate: for $v_{\circ}$ to go through $d S$, it is necessary that

$$
\sigma=\sigma_{c}=\frac{v-v_{\#}}{\left|v-v_{\#}\right|}
$$




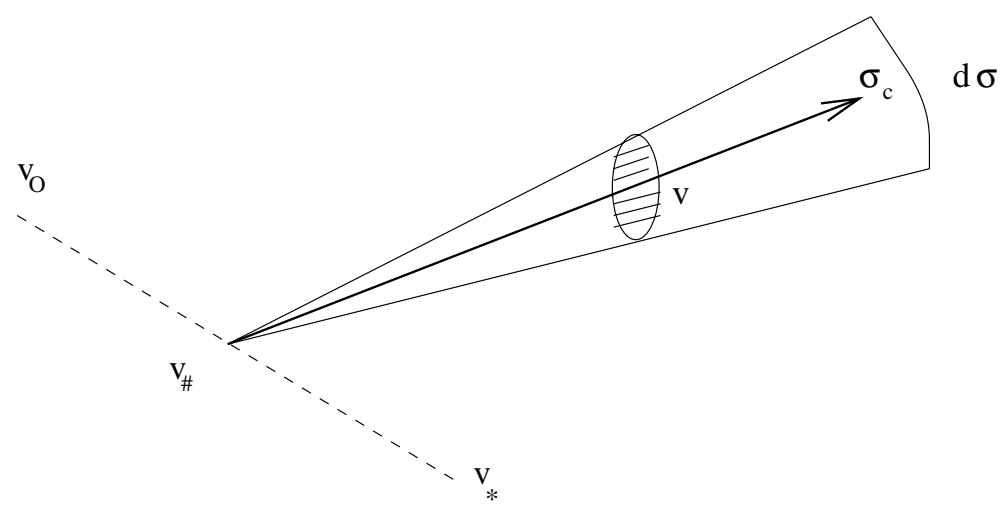

FigurE 4. Second step of the collisional scheme

$I^{+}$corresponds to $\left(\sigma_{c}\right)_{1}>0$, and $I^{-}$to $\left(\sigma_{c}\right)_{1}<0$; moreover, viewed from $v_{\#}, d \sigma=d S\left|\left(\sigma_{c}\right)_{1}\right| /\left|v-v_{\#}\right|^{N-1}$. Therefore, we obtain

$$
j^{(b)}=\int d v_{\circ} d v_{*} f\left(v_{\circ}\right) f\left(v_{*}\right) \widetilde{B}\left(v_{\circ}-v_{*}, \sigma_{c}\right) \frac{\sigma_{c}}{\left|v-v_{\#}\right|^{N-1}} \cdot
$$

By the change of variables $\left(v_{\circ}, v_{*}\right) \rightarrow\left(\rho, \sigma, w_{*}\right)$ with

$$
\left\{\begin{array}{l}
v_{\#}=v-\rho \sigma \\
v_{\circ}-v_{*}=v-w_{*},
\end{array}\right.
$$

with Jacobian $\rho^{N-1}$, we get

$$
j^{(b)}=\int d w_{*} d \sigma \widetilde{B}\left(v-w_{*}, \sigma\right) \int_{0}^{\frac{\left|v-w_{*}\right|}{2}} d \rho f\left(v-\rho \sigma-\frac{v-w_{*}}{2}\right) f\left(v-\rho \sigma+\frac{v-w_{*}}{2}\right) \sigma .
$$

Moreover, in these variables, by (5) the postcollisional velocity of $P_{\circ}$ is

$$
v_{\circ}^{\prime}=v+\left(\frac{\left|v-w_{*}\right|}{2}-\rho\right) \sigma
$$

so that the flux for the collisional invariant $C$ is

$$
\begin{array}{r}
j^{(b)}(C)=\int d w_{*} d \sigma \widetilde{B}\left(v-w_{*}, \sigma\right) \int_{0}^{\frac{\left|v-w_{*}\right|}{2}} d \rho f\left(v-\rho \sigma-\frac{v-w_{*}}{2}\right) \\
f\left(v-\rho \sigma+\frac{v-w_{*}}{2}\right) C\left(v+\left(\frac{\left|v-w_{*}\right|}{2}-\rho\right) \sigma\right) \sigma .
\end{array}
$$


We now compute the divergence of the opposite of (27), after the change of variables

$$
\begin{aligned}
& -\int d w_{*} d \sigma \widetilde{B}\left(v-w_{*}, \sigma\right) \int_{0}^{\frac{\left|v-w_{*}\right|}{2}} d \rho f\left(v-\rho \sigma-\frac{v-w_{*}}{2}\right) f\left(v-\rho \sigma+\frac{v-w_{*}}{2}\right) \sigma \\
= & -\int d w d \sigma \widetilde{B}(w, \sigma) \int_{0}^{\frac{|w|}{2}} d \rho f\left(v-\rho \sigma-\frac{w}{2}\right) f\left(v-\rho \sigma+\frac{w}{2}\right) \sigma .
\end{aligned}
$$

Thus,

$$
\begin{aligned}
-\nabla \cdot j^{(b)} & =-\int d w d \sigma \widetilde{B}(w, \sigma) \int_{0}^{\frac{|w|}{2}} d \rho \sigma \cdot \nabla\left[f\left(v-\rho \sigma-\frac{w}{2}\right) f\left(v-\rho \sigma+\frac{w}{2}\right)\right] \\
& =\int d w d \sigma \widetilde{B}(w, \sigma) \int_{0}^{\frac{|w|}{2}} d \rho \partial_{\rho}\left[f\left(v-\rho \sigma-\frac{w}{2}\right) f\left(v-\rho \sigma+\frac{w}{2}\right)\right] \\
& =\int d w d \sigma \widetilde{B}(w, \sigma)\left[f\left(v-\rho \sigma-\frac{w}{2}\right) f\left(v-\rho \sigma+\frac{w}{2}\right)\right]_{\rho=0}^{\rho=\frac{|w|}{2}} \\
& =-\int d w d \sigma \widetilde{B}(w, \sigma) f\left(v-\frac{w}{2}\right) f\left(v+\frac{w}{2}\right) \\
& \quad+\int d w d \sigma \widetilde{B}(w, \sigma) f\left(v-\frac{|w|}{2} \sigma-\frac{w}{2}\right) f\left(v-\frac{|w|}{2} \sigma+\frac{w}{2}\right) \\
& \equiv-(\mathrm{Ib})+(\mathrm{IIb}) .
\end{aligned}
$$

By a spherical change of coordinates $w=r \beta$, the last integral (IIb) is

$$
\begin{aligned}
& \int d \beta d r d \sigma r^{N-1} \widetilde{B}(r \beta, \sigma) f\left(v-\frac{r}{2} \sigma-\frac{r}{2} \beta\right) f\left(v-\frac{r}{2} \sigma+\frac{r}{2} \beta\right) \\
= & \int d \beta d r d \sigma r^{N-1} \widetilde{B}(r \sigma, \beta) f\left(v-\frac{r}{2} \sigma-\frac{r}{2} \beta\right) f\left(v-\frac{r}{2} \sigma+\frac{r}{2} \beta\right)
\end{aligned}
$$

since $B(r \beta, \sigma)=B(r \sigma, \beta)$. Now, it suffices to proceed to the change of variables $v-(r / 2) \sigma=\left(v+v_{*}\right) / 2$, i.e. $v_{*}=v-r \sigma$, to obtain

$$
\int d v_{*} d \beta \widetilde{B}\left(v-v_{*}, \beta\right) f\left(\frac{v+v_{*}}{2}-\frac{\left|v-v_{*}\right|}{2} \beta\right) f\left(\frac{v+v_{*}}{2}+\frac{\left|v-v_{*}\right|}{2} \beta\right),
$$

which is the gain term of Boltzmann's collision operator. Moreover, (Ib) and (Ia) are equal, as can be seen by setting $w=2 \rho \beta$ in (Ib).

The same computation with $C(v)=v_{k}$ for instance leads to

$$
\begin{aligned}
-\nabla \cdot j^{(b)}\left(v_{k}\right)=-\int d w d \sigma \widetilde{B}(w, \sigma) f\left(v-\frac{w}{2}\right) f & \left(v+\frac{w}{2}\right)\left(v_{k}+\frac{|w|}{2} \sigma_{k}\right) \\
& +\int d w d \sigma \widetilde{B}(w, \sigma) f\left(v-\frac{|w|}{2} \sigma-\frac{w}{2}\right) f\left(v-\frac{|w|}{2} \sigma+\frac{w}{2}\right) v_{k} .
\end{aligned}
$$

Since, by $\sigma \rightarrow-\sigma$

$$
-\int d w d \sigma \widetilde{B}(w, \sigma) f\left(v-\frac{w}{2}\right) f\left(v+\frac{w}{2}\right) \frac{|w|}{2} \sigma_{k}=0
$$

we obtain the desired result. 
As a conclusion, summing up the contributions of steps 1 and 3 , we find in particular that $Q=-\nabla \cdot J$ and $Q v_{k}=-\nabla \cdot J^{k}$ with

$$
\begin{aligned}
J=-\int d v_{*} d \sigma \widetilde{B}\left(v-v_{*}, \sigma\right) \int_{0}^{\frac{\left|v-v_{*}\right|}{2} d r\left\{f\left(v+r \frac{v-v_{*}}{\left|v-v_{*}\right|}\right) f\left(v_{*}+r \frac{v-v_{*}}{\left|v-v_{*}\right|}\right) \frac{v-v_{*}}{\left|v-v_{*}\right|}\right.} \\
\left.-f\left(v-r \sigma-\frac{v-v_{*}}{2}\right) f\left(v-r \sigma+\frac{v-v_{*}}{2}\right) \sigma\right\}
\end{aligned}
$$

$$
\begin{aligned}
& J^{k}=-\int d v_{*} d \sigma \widetilde{B}\left(v-v_{*}, \sigma\right) \int_{0}^{\frac{\left|v-v_{*}\right|}{2}} d r \\
& \left\{f\left(v+r \frac{v-v_{*}}{\left|v-v_{*}\right|}\right) f\left(v_{*}+r \frac{v-v_{*}}{\left|v-v_{*}\right|}\right) \frac{v-v_{*}}{\left|v-v_{*}\right|}\left(v_{k}+r \frac{\left(v-v_{*}\right)_{k}}{\left|v-v_{*}\right|}\right)\right. \\
& \left.-f\left(v-r \sigma-\frac{v-v_{*}}{2}\right) f\left(v-r \sigma+\frac{v-v_{*}}{2}\right) \sigma\left(v_{k}+\left(\frac{\left|v-v_{*}\right|}{2}-r\right) \sigma_{k}\right)\right\} .
\end{aligned}
$$

The expression of $A_{i k}=J_{i}^{k}-J_{i} v_{k}$ follows.

\section{LANDAU'S COLLISION OPERATOR}

To write Landau's collision operator as a double divergence, we first note that

$$
\left(a_{i j} * f\right) \partial_{j} f-\left(b_{i} * f\right) f=\partial_{j}\left(\left(a_{i j} * f\right) f\right)-2\left(b_{i} * f\right) f,
$$

hence it suffices to prove that for each $i,\left(b_{i} * f\right) f$ is a divergence. After the previous study, it is easy to guess a convenient expression,

$$
\begin{aligned}
& -\int d v_{*} b\left(v-v_{*}\right) f(v) f\left(v_{*}\right) \\
& =-\int d R d \beta R^{N-1} b(-R \beta) f(v) f(v+R \beta) \\
& =\int d R d \beta\left[(R-\rho)^{N-1} b(\rho \beta-R \beta) f(v+\rho \beta) f(v+R \beta)\right]_{\rho=0}^{\rho=R} \\
& =\int d R d \beta \int_{0}^{R} d \rho \partial_{\rho}\left[(R-\rho)^{N-1} b(\rho \beta-R \beta) f(v+\rho \beta) f(v+R \beta)\right] \\
& =\int d R d \beta \int_{0}^{R} d \rho\left\{\left(-\partial_{R}\right)\left[(R-\rho)^{N-1} b(\rho \beta-R \beta)\right] f(v+\rho \beta) f(v+R \beta)\right. \\
& \left.\quad \quad+(R-\rho)^{N-1} b(\rho \beta-R \beta) \partial_{\rho}[f(v+\rho \beta)]\right\} \\
& =\int d R d \beta \int_{0}^{R} d \rho\left\{(R-\rho)^{N-1} b(\rho \beta-R \beta) f(v+\rho \beta) \partial_{R}[f(v+R \beta)]\right. \\
& \left.\quad \quad+(R-\rho)^{N-1} b(\rho \beta-R \beta) \partial_{\rho}[f(v+\rho \beta)]\right\} \\
& =\int d R d \beta \int_{0}^{R} d \rho(R-\rho)^{N-1} b(\rho \beta-R \beta) \beta \cdot \nabla(f(v+\rho \beta) f(v+R \beta)) \\
& =\nabla \cdot\left(\int d R d \beta \int_{0}^{R} d \rho(R-\rho)^{N-1} b(\rho \beta-R \beta) f(v+\rho \beta) f(v+R \beta) \beta\right),
\end{aligned}
$$


checking that all boundary terms vanish when integrating by parts. We note that we did not use the fact that $b$ is odd, which is the condition for $\int d v d v_{*} b\left(v-v_{*}\right) f(v) f\left(v_{*}\right)$ to be vanishing in the general case. This simply means that the above flux does not necessarily vanish at infinity.

Another simpler possibility to compute a convenient flux is to look for it in the form

$$
\int d v_{\circ} d v_{*} f\left(v_{*}\right) f\left(v_{\circ}\right) D\left(v, v_{\circ}, v_{*}\right)
$$

and to choose

$$
D\left(v, v_{\circ}, v_{*}\right)=b\left(v_{\circ}-v_{*}\right) \nabla U\left(v-v_{\circ}\right)
$$

where $U$ is the fundamental solution of the Laplace operator in $\mathbb{R}^{N}$.

\section{The Asymptotics of GRAZING COLLISIONS}

As an application of the conservative form of Boltzmann's operator, we give an adaptation of Landau's original argument to pass from (1) to (7). We consider a sequence of kernels concentrating on grazing collisions, i.e. for example

$$
\Psi_{\varepsilon}(|z|) \equiv \frac{\pi}{2} \int_{S^{N-1}} d \omega B_{\varepsilon}(z, \omega)(z, \omega)^{2}=\Psi(|z|) \text { (independently of } \varepsilon \text { ). }
$$

In the limit, only the collisions with $\left(v-v_{*}, \omega\right) \simeq 0$ are taken into account. We denote by $Q_{\varepsilon}$ the Boltzmann-type collision operator associated with $B_{\varepsilon}$. It was proven by Desvillettes [2] that if (say) $f \in W^{3, \infty}$ and has compact support, then $Q_{\varepsilon}(f, f) \longrightarrow Q_{L}(f, f)$ in $L_{\text {loc }}^{1}$ as $\varepsilon$ goes to 0 . Here we shall content to show how one can simply prove that

$$
-J_{\varepsilon}(f, f) \underset{\varepsilon \rightarrow 0}{\longrightarrow} \int_{\mathbb{R}^{N}} d v_{*} a_{i j}\left(v-v_{*}\right)\left(f_{*} \frac{\partial f}{\partial v_{i}}-f \frac{\partial f_{*}}{\partial v_{* i}}\right) .
$$

We note that under very weak assumptions $Q_{\varepsilon}(f, f) \longrightarrow Q_{L}(f, f)$ in the sense of distributions, see for example [6]. A direct proof may however be useful to get strong convergence once a priori bounds are known.

Starting from

$$
-J_{\varepsilon}=\int_{\left(v-v_{*}, \omega\right)>0} d v_{*} d \omega B_{\varepsilon}\left(v-v_{*}, \omega\right) \int_{0}^{\left(v-v_{*}, \omega\right)} d r f(v+r \omega) f\left(v_{*}+r \omega\right) \omega
$$

we note that $\omega \rightarrow-\omega, r \rightarrow-r$ leaves the integrand invariant and transforms $\left(v-v_{*}, \omega\right)>0$ into $\left(v-v_{*}, \omega\right)<0$; therefore,

$$
-J_{\varepsilon}=\frac{1}{2} \int d v_{*} d \omega B_{\varepsilon}\left(v-v_{*}, \omega\right) \int_{0}^{\left(v-v_{*}, \omega\right)} d r f(v+r \omega) f\left(v_{*}+r \omega\right) \omega
$$

with the convention that $\int_{a}^{b} g=-\int_{b}^{a} g$. We assume for instance $N=3$. For given $v, v_{*}$, we represent $\omega$ by spherical coordinates $\theta, \phi$, and for given $\phi$ we denote by $\left(\frac{v-v_{*}}{\left|v-v_{*}\right|}\right)^{\perp}$ the unit vector orthogonal to $v-v_{*}$, with angle $\phi$. Then we write

$$
\omega=\left(\frac{v-v_{*}}{\left|v-v_{*}\right|}\right)^{\perp}+\left(\frac{v-v_{*}}{\left|v-v_{*}\right|}, \omega\right) \frac{v-v_{*}}{\left|v-v_{*}\right|}+O\left(\left(\frac{v-v_{*}}{\left|v-v_{*}\right|}, \omega\right)^{2}\right),
$$


and accordingly,

$$
\begin{aligned}
&-J_{\varepsilon}=\frac{1}{2} \int d v_{*} d \omega B_{\varepsilon}\left(v-v_{*}, \omega\right) \int_{0}^{\left(v-v_{*}, \omega\right)} d r f(v+r \omega) f\left(v_{*}+r \omega\right)\left(\frac{v-v_{*}}{\left|v-v_{*}\right|}\right)^{\perp} \\
&+\frac{1}{2} \int d v_{*} d \omega B_{\varepsilon}\left(v-v_{*}, \omega\right) \int_{0}^{\left(v-v_{*}, \omega\right)} d r f(v+r \omega) f\left(v_{*}+r \omega\right)\left(\frac{v-v_{*}}{\left|v-v_{*}\right|}, \omega\right) \frac{v-v_{*}}{\left|v-v_{*}\right|} \\
&+\frac{1}{2} \int d v_{*} d \omega B_{\varepsilon}\left(v-v_{*}, \omega\right) \int_{0}^{\left(v-v_{*}, \omega\right)} d r f(v+r \omega) f\left(v_{*}+r \omega\right) O\left(\left(\frac{v-v_{*}}{\left|v-v_{*}\right|}, \omega\right)^{3}\right) .
\end{aligned}
$$

Assuming $f$ to be $C^{2}$,

$$
\begin{gathered}
f\left(v_{*}+r \omega\right) f(v+r \omega)=f(v) f\left(v_{*}\right)+r f\left(v_{*}\right) \nabla f(v) \cdot \omega+r f(v) \nabla f\left(v_{*}\right) \cdot \omega+O\left(r^{2}\right) \\
-J_{\varepsilon}=\frac{1}{2} \int d v_{*} d \omega B_{\varepsilon}\left(v-v_{*}, \omega\right)\left(v-v_{*}, \omega\right) f f_{*}\left(\frac{v-v_{*}}{\left|v-v_{*}\right|}\right)^{\perp} \\
+\frac{1}{2} \int d v_{*} d \omega B_{\varepsilon}\left(v-v_{*}, \omega\right) \frac{\left(v-v_{*}, \omega\right)^{2}}{2}\left(f \nabla_{*} f_{*}+f_{*} \nabla f\right) \cdot\left(\frac{v-v_{*}}{\left|v-v_{*}\right|}\right)^{\perp} \\
+\frac{1}{2} \int d v_{*} d \omega B_{\varepsilon}\left(v-v_{*}, \omega\right)\left(v-v_{*}, \omega\right)^{2} f f_{*} \frac{v-v_{*}}{\left|v-v_{*}\right|^{2}} \\
+\frac{1}{2} \int d v_{*} d \omega B_{\varepsilon}\left(v-v_{*}, \omega\right) O\left(\left(\frac{v-v_{*}}{\left|v-v_{*}\right|}, \omega\right)^{3}\right) 1_{\left|\left(v-v_{*}, \omega\right)\right| \leq K} \\
+\frac{1}{2} \int d v_{*} d \omega B_{\varepsilon}\left(v-v_{*}, \omega\right) f(v+r \omega) f\left(v_{*}+r \omega\right)\left|\left(v-v_{*}, \omega\right)\right| 1_{\left|\left(v-v_{*}, \omega\right)\right| \geq K} .
\end{gathered}
$$

Under convenient assumptions on $f$ and $B$, the last two terms vanish in the limit $\varepsilon \rightarrow 0$, since

$$
\int d \omega B_{\varepsilon}\left(v-v_{*}, \omega\right)\left(\frac{v-v_{*}}{\left|v-v_{*}\right|}, \omega\right)^{3} \longrightarrow 0
$$

Moreover, by symmetry,

$$
\frac{1}{2} \int d v_{*} d \omega B_{\varepsilon}\left(v-v_{*}, \omega\right)\left(v-v_{*}, \omega\right) f f_{*}\left(\frac{v-v_{*}}{\left|v-v_{*}\right|}\right)^{\perp}=0
$$

Therefore, the limit is that of

$$
\begin{aligned}
\frac{1}{2} \int d v_{*} d \omega B_{\varepsilon}\left(v-v_{*}, \omega\right) \frac{\left(v-v_{*}, \omega\right)^{2}}{2}\left(f \nabla_{*} f_{*}+f_{*} \nabla f\right) \cdot\left(\frac{v-v_{*}}{\left|v-v_{*}\right|}\right)^{\perp} & \\
& +\frac{1}{2} \int d v_{*} d \omega B_{\varepsilon}\left(v-v_{*}, \omega\right)\left(v-v_{*}, \omega\right)^{2} f f_{*} \frac{v-v_{*}}{\left|v-v_{*}\right|^{2}}
\end{aligned}
$$

Then we write $\omega$ in spherical coordinates with axis $v-v_{*}$ : let $\theta, \phi$ be the corresponding angles, we set (for instance) $B\left(v-v_{*}, \omega\right)=\mu(\theta) C\left(v-v_{*}\right)$. To conclude that the limit is the right-hand side of (31), it suffices to use (30) and the (classical) identities $(c f .[2,6])$ 


$$
\begin{gathered}
\int_{0}^{2 \pi} d \phi\left(f \nabla_{*} f_{*}+f_{*} \nabla f\right) \cdot\left(\frac{v-v_{*}}{\left|v-v_{*}\right|}\right)^{\perp}(\phi)=\pi \int \Pi\left(v-v_{*}\right) \cdot\left(f_{*} \nabla f+f \nabla_{*} f_{*}\right), \\
\nabla \cdot\left(\Pi(z)|z|^{2} C(z)\right)=-2 z C(z) .
\end{gathered}
$$

The author thanks F. Golse for asking him the question which is at the basis of this work.

\section{REFERENCES}

[1] C. Cercignani, The Boltzmann equation and its applications. Springer, New York (1988).

[2] L. Desvillettes, On asymptotics of the Boltzmann equation when the collisions become grazing. Transp. Theory Stat. Phys. 21 (1992) 259-276.

[3] M. Lemou, private communication.

[4] E.M. Lifchitz and L.P. Pitaevskii, Physical Kinetics - Course in theoretical physics, Vol. 10. Pergamon, Oxford, (1981).

[5] J.C. Maxwell, On the dynamical theory of gases. Phil. Trans. R. Soc. Lond. 157 (1886) 49-88.

[6] C. Villani, On a new class of weak solutions to the spatially homogeneous Boltzmann and Landau equations. Arch. Rat. Mech. Anal. 143 (1997) 273-307. 